outcome supports timely management of children using an integrated care-pathway led by a multidisciplinary team. The vast majority of children may be safely managed without surgery.

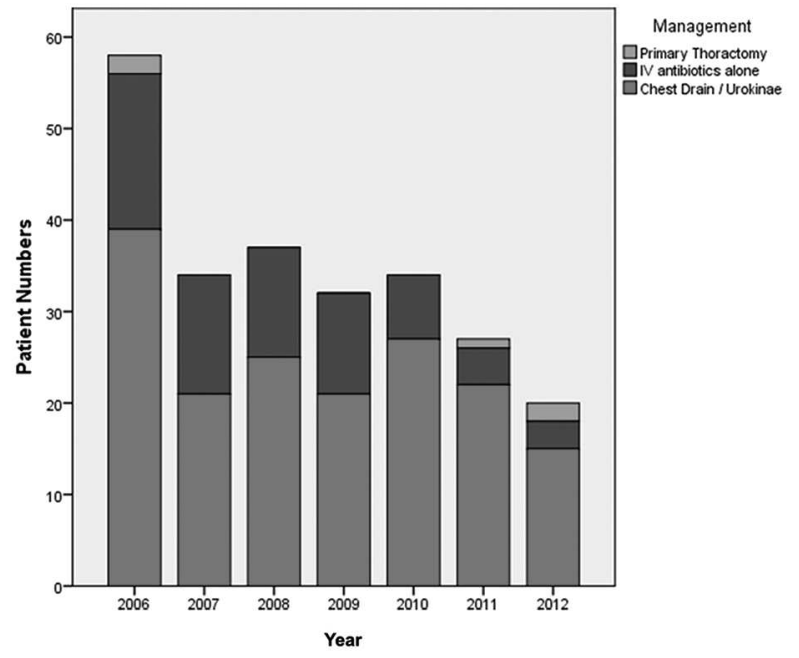

Abstract S77 Figure 1. Number of inpatient admissions and primary management by year.

\section{Managing pleural effusions}

\section{S78 EVALUTION OF AN AMBULATORY PLEURAL SERVICE: COSTS AND BENEFITS}

${ }^{1} \mathrm{RL}$ Young, ${ }^{2} \mathrm{R}$ Bhatnagar, ${ }^{3} \mathrm{ZD}$ Mason, ${ }^{4} \mathrm{AJ}$ Benson, ${ }^{5} \mathrm{CE}$ Hooper, ${ }^{2} \mathrm{AO}$ Clive, ${ }^{6} \mathrm{~N}$ ZahanEvans, ${ }^{6}$ AJ Morley, ${ }^{6}$ JE Harvey, ${ }^{6}$ ARL Medford, ${ }^{2}$ NA Maskell; ${ }^{1}$ University of Bristol Medical School, Bristol, United Kingdom; ' University of Bristol Academic Respiratory Unit, Bristol, United Kingdom; ${ }^{3}$ Department of Clinical Coding, North Bristol NHS Trust, Bristol, United Kingdom; ${ }^{4}$ Finance Department, North Bristol NHS Trust, Bristol, United Kingdom; ${ }^{5}$ Worcestershire Acute Hospitals NHS Trust, Worcester, United Kingdom; ${ }^{6}$ North Bristol Lung Centre, North Bristol NHS Trust, Bristol, United Kingdom

10.1136/thoraxjnl-2013-204457.85

Background Outpatient management of undiagnosed pleural effusions is increasing. Payment for managing these patients is usually based on standard outpatient Healthcare Resource Group (HRG) codes. For 2013/14, a new Best Practice Tariff (BPT) of $£ 1534$ has been introduced to further disincentivize emergency inpatient management. We audited our service and examined what effects this tariff may have when applied.
Methods Our well-established tertiary pleural service serves a local population of around 540,000. New patients are seen in a weekly pleural clinic or in a daily respiratory admission avoidance (Hot) clinic, which had standard 2012/13 tariffs of $£ 223$ and $£ 334$ respectively. The service sees approximately 150 new effusion patients per year in clinic and 3 new patients per week in Hot. Around 50 medical thoracoscopies and 60 indwelling pleural catheter insertions are performed each year.

We audited randomly selected patients from our large, prospectively-maintained database. All audited patients were seen as new pleural effusion referrals between 2008 and 2012. Diagnosis was confirmed after a minimum of 12 months' follow-up.

Results 146 patients were audited. Median age 76(range 21-93), $71 \%$ male. Final diagnoses were mesothelioma $(n=28,19 \%)$, lung cancer $(\mathrm{n}=10,7 \%)$, breast cancer $(\mathrm{n}=13,9 \%)$, other cancer $(\mathrm{n}=31,21 \%)$, pleural infection $(\mathrm{n}=15,10 \%)$, benign pleuritis $(\mathrm{n}=11,8 \%)$ and other $(\mathrm{n}=38,26 \%) .92 \%$ of patients avoided direct admission following their initial clinic appointment.

115 patients $(79 \%)$ underwent ultrasound-guided pleural aspiration at their initial appointment and 63(43\%) patients underwent subsequent pleural biopsy. For patients with malignancy, diagnostic sensitivity on first fluid cytology was 27\% (adenocarcinoma $\mathrm{n}=15,80 \%$; mesothelioma $\mathrm{n}=21,5 \%)$, and $93 \%(25 /$ 27) for medical thoracoscopy biopsy. Histological/cytological diagnosis took a median of 20 days (IQR 10-33) from presentation. There were no significant procedural complications noted (bleeding, pneumothorax, empyema). 97\%(58/60) of patients surveyed rated the service as either very good or excellent.

Conclusions Ambulatory management of undiagnosed effusions is efficacious, avoids hospitalisation in the vast majority and is preferred by patients. The 2013/14 pleural effusion BPT promotes admission avoidance by encouraging appropriate outpatient management. Trust reimbursement for practising in this way should facilitate enough resource to enable new pleural services to be established where required.

\section{S79 COMPARING THE QUALITY OF LIFE AND COST- EFFECTIVENESS OF INDWELLING PLEURAL CATHETER VS. TALC PLEURODESIS FOR MALIGNANT PLEURAL EFFUSIONS}

${ }^{1} \mathrm{E}$ Penz, ${ }^{2} \mathrm{E}$ Mishra, ${ }^{3} \mathrm{H}$ Davies, ${ }^{1} \mathrm{~B}$ Manns, ${ }^{4} \mathrm{RF}$ Miller, ${ }^{2} \mathrm{~N}$ Rahman; ${ }^{1}$ University of Calgary, Calgary, Canada; ${ }^{2}$ Oxford University, Oxford, United Kingdom; ${ }^{3}$ University Hospital of Wales, Cardiff, United Kingdom; ${ }^{4}$ University College London, London, United Kingdom

10.1136/thoraxjnl-2013-204457.86

Abstract S78 Table 1. Estimates of income using old and new tariffs based on yearly patient numbers and audit data

\begin{tabular}{|c|c|c|c|c|}
\hline Type of encounter & Number & 2012 - 2013tariff & 2013 - 2014 BPT tariff & Notes \\
\hline $\begin{array}{l}\text { Outpatient/Hot } \\
\text { new effusion }\end{array}$ & 300 & $\begin{array}{l}{[300 *(223+334 / 2)]=} \\
\mathrm{f} 83,550 * 0.92= \\
\mathrm{f} 76,866\end{array}$ & $\begin{array}{l}\left.\text { a. }\left[300^{*} 0.70\right)^{*} 1534\right] \\
=f 322,140 * 0.92 \\
=f 296,369 \\
\text { b. }\left[\left(300^{*} 0.3\right)^{*}(189+329 / 2)\right] \\
=f 23,310^{*} .92 \\
=f 21,445 a+b=f 317,814\end{array}$ & $\begin{array}{l}\text { Assumed } 70 \% \text { of patients undergo aspiration at first } \\
\text { appointment (therefore eligible } \\
\text { for BPT), and } 8 \% \text { need admission (therefore } \\
\text { not billed as outpatients) }\end{array}$ \\
\hline Subsequent aspiration & 60 & $60 * 544=f 32,640$ & $18 * 544=\mathrm{f} 9792$ & Assumed $20 \%$ go on to have aspiration later \\
\hline IPC insertion & 60 & $60 * 544=£ 32,640$ & $60 * 544=£ 32,640$ & All IPCs inserted as day case \\
\hline Medical thoracoscopy & 50 & $\begin{array}{l}50^{*}[(544+2153) / 2] \\
=\mathrm{f} 67,425\end{array}$ & $50^{*}[(544+2153) / 2]=f 67,425$ & Assumed $50 \%$ of patients receive talc \\
\hline 12 MONTH TOTAL & & $f 209,571$ & $£ 427,671$ & Extra incomef218,100 \\
\hline
\end{tabular}

CODES \& TARIFFS: Diagnostic thoracoscopy (T11.1, HRG DZ06Z, f544); Thoracoscopy with talc (T10.2, HRG DZ04B, f2153); IPC insertion (T12.4, HRG DZ06Z, f544); Aspiration (T12.3, HRG DZ06Z, f544); Respiratory o/p first attendance (WF01B, f223 in 12/13, f189 in 13/14); HOT clinic (locally agreed f334 in 12/13, f329 in 13/14). BPT aspirations must occur on elective list after initial assessment. 